\title{
Optimal Laser Heating of Plasmas with Constant Density ${ }^{1}$
}

\author{
J. E. VITELA ${ }^{2}$ AND A. Z. AKCASU \\ Communicated by C. T. Leondes
}

\begin{abstract}
The laser heating of a plasma with constant density is analyzed using optimal control theory. Heating strategies that minimize the total energy spent, the heating time, or a linear combination of the two, for several values of weighting coefficients, are obtained by determining the optimal laser intensity associated with each point of the phase plane. A numerical example is used to illustrate the application of the theory. In this particular example, savings in the energy spent up to $75 \%$, compared with the energy required using a constant laser pulse, are obtained when minimum energy trajectories are implemented. Strategies that minimize the heating time, however, did not yield a significant reduction in the heating time. Numerical results may depend strongly on the initial state of the system as well as on the final ion temperature of the plasma.
\end{abstract}

Key Words. Optimal control theory, plasmas, laser heating, singular controls.

\section{Introduction}

This paper is concerned with the optimal heating of plasmas by a laser beam, in order to minimize the total laser energy required to increase the ion temperature to a desired value, within acceptable heating times. The optimization problem arises because the laser energy is first absorbed by the electrons, increasing thus the electron temperature. Overheating the

\footnotetext{
${ }^{1}$ Based on a thesis submitted as partial fulfillment for a Ph.D. degree at the University of Michigan. Financial support, provided to the first author by Consejo Nacional de Ciencia y Tecnología, Universidad Nacional Autónoma de México, by the National Science Foundation, and by the Michigan Memorial Phoenix Project, is deeply appreciated.

${ }^{2}$ Researcher, Centro de Estudios Nucleares, Universidad Nacional Autónoma de México, 04510 México, D.F., Mexico.

${ }^{3}$ Professor, Department of Nuclear Engineering, University of Michigan, Ann Arbor, Michigan 48109.
} 
electrons reduces the electron-ion energy transfer rate and the coupling of the laser beam to the electrons, while increasing the radiation losses. Furthermore, certain types of instabilities may be excited (Ref. 1), if the difference between the electron and ion temperatures becomes too large. Hence, the application of the maximum laser intensity available may not always be desirable. This suggests heating strategies by modulating the laser intensity during heating, to achieve various conflicting objectives. For example, one may try to maximize the electron-ion energy transfer rate to minimize the heating time, or one may want to minimize the total energy necessary to heat the ions by maximizing the coupling between the laser beam and the electrons, while minimizing the bremsstrahlung losses.

In 1975, Vagners et al (Ref. 2) solved the problem of minimum ion heating time in a plasma confined in a strong solenoidal magnetic field, neglecting bremsstrahlung radiation losses and the work done by the plasma during expansion against the magnetic field. They assumed a given total laser energy available for heating the plasma ions and determined the optimal laser intensity profile that minimizes the heating time for only one set of initial conditions.

In this paper, we extend their work by including bremsstrahlung radiation losses but assuming constant plasma density. Here, we do not constrain the total energy spent during the heating time, since this may lead to cases with no solution; instead, we minimize a linear combination of the total energy spent and the heating time with appropriate weighting coefficients. By adjusting the latter, one may obtain physically reasonable heating time and total laser energy within permissible limits.

The solution of the problem described above requires the use of optimal control theory (Refs. 3 and 4). To give an idea about the type of information one obtains by resorting to optimal control, we pose the following question: A fully ionized plasma is produced initially in equilibrium at $10 \mathrm{eV}$ with a density of $2 \times 10^{17} / \mathrm{cm}^{3}$. It is desired to raise the ion temperature to $5 \mathrm{KeV}$ using a $\mathrm{CO}_{2}$ laser, with a maximum intensity $I_{0}=2.25 \times 10^{36} \mathrm{eV} / \mathrm{cm}^{2} \mathrm{~s}$, (a) in a minimum time, (b) with minimum laser energy. If the full intensity is used, the heating time is $T=101.65 \mu \mathrm{s}$ and the total energy is $E=$ $22.87 \times 10^{25} \mathrm{eV} / \mathrm{cm}^{2}$. When the heating time is minimized, one finds (see Table 1) $T=100.75 \mu \mathrm{s}$ and $E=22.13 \times 10^{25} \mathrm{eV} / \mathrm{cm}^{2}$, with a little gain in the total laser energy. However, when the total laser energy is minimized one finds $T=771.17 \mu \mathrm{s}$ and $E=5.18 \times 10^{25} \mathrm{eV} / \mathrm{cm}^{2}$, with a saving of the order of $75 \%$ in laser energy at the expense of heating time. Figure 1 shows the time dependence of the optimal laser intensity in the above two cases. By minimizing a linear combination of the heating time and the total energy, we obtain heating strategies leading to significant savings in the total laser energy requirement with a small increase in the heating time. For example, 
Table 1. Heating data corresponding to the optimal trajectories in Fig. 10.

\begin{tabular}{ccccccc}
\hline$C_{1} / C_{2} I_{0}$ & $\begin{array}{c}\text { Switching } \\
\text { time } \\
U_{\max } \rightarrow U_{s} \\
(\mathrm{ps})\end{array}$ & $\begin{array}{c}\text { Switching } \\
\text { time } \\
U_{s} \rightarrow U_{\max } \\
(\mu \mathrm{s})\end{array}$ & $\begin{array}{c}\text { Switching } \\
\text { time } \\
U_{\mathrm{s}} \rightarrow U_{\min } \\
(\mu \mathrm{s})\end{array}$ & $\begin{array}{c}\text { Switching } \\
\text { time } \\
U_{\max } \rightarrow U_{\min } \\
(\mu \mathrm{s})\end{array}$ & $\begin{array}{c}\text { Heating } \\
\text { time } \\
(\mu \mathrm{s})\end{array}$ & $\begin{array}{c}\text { Heating } \\
\text { energy } \\
\left(10^{25} \mathrm{eV} / \mathrm{cm}^{2}\right)\end{array}$ \\
\hline 0 & 0.02 & - & 695.87 & - & 771.17 & 5.818 \\
$10^{-4}$ & 1.62 & - & 617.49 & - & 692.60 & 5.826 \\
$1 / 5$ & 28.4 & - & 98.36 & - & 147.15 & 8.216 \\
$1 / 2$ & 30.1 & - & 79.53 & - & 124.58 & 9.811 \\
1 & 30.1 & - & 70.62 & - & 113.82 & 11.51 \\
2 & 31.1 & 21.84 & - & 69.90 & 106.97 & 13.66 \\
5 & 31.2 & 7.99 & - & 78.32 & 102.70 & 16.57 \\
$\infty$ & 31.3 & 3.26 & - & - & 100.75 & 22.13 \\
Constant pulse & - & - & - & - & 101.65 & 22.87 \\
\hline
\end{tabular}

in one of the cases in Table 1, we found $T=113.8 \mu \mathrm{s}$ and a total energy of $E=11.51 \times 10^{25} \mathrm{eV} / \mathrm{cm}^{2}$, implying $50 \%$ saving in energy with only $11 \%$ increase in the heating time.

This paper provides a complete solution of the optimal laser heating problem, for the plasma model described below, by obtaining the optimal laser intensity profile for any initial and final state.

\section{Mathematical Description of Plasma Heating}

We assume that the laser radiation illuminates the plasma column uniformly and that electrons and ions are always Maxwellian with uniform temperature $T_{e}(t)$ and $T_{i}(t)$, respectively, and constant densities $n_{e}=n_{i}=n$.

In underdense plasmas (plasmas with densities less than that in which the laser frequency equals the plasma frequency), the index of refraction is less than one and increases in regions of lower plasma densities. Hence, the laser light will be refracted toward low-density regions of the plasma (Ref. 5). By assuming, as we mentioned earlier, that the laser radiation illuminates the plasma column uniformly, we virtually neglect this effect at the outset. In addition, the laser frequency is taken to be greater than the plasma frequency so that reflection losses at the vacuum plasma boundary are minimal.

As pointed out by Yuen et al (Ref. 6), in the process of laser heating of fully ionized plasmas, the laser energy is mainly absorbed by the electrons; and the dominant absorption mechanism is that of inverse bremsstrahlung as shown by Dawson et al (Ref. 7). 

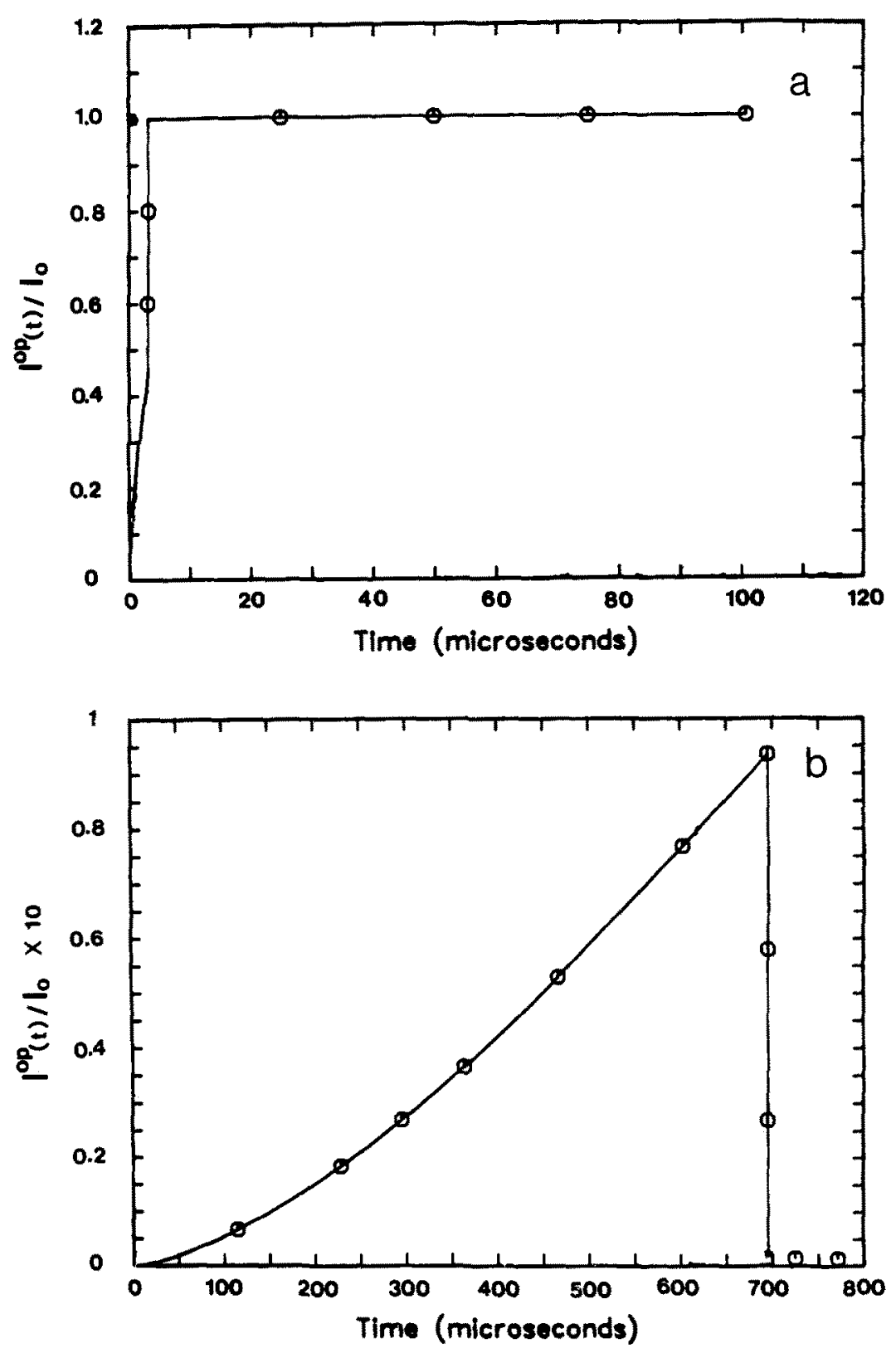

Fig. 1. Top: Laser pulse that minimizes the heating time. Bottom: Laser pulse that minimizes the heating energy. 
The equations describing the laser heating of the plasma under the above assumptions are the following:

$$
\begin{aligned}
& (3 / 2)(d / d t)\left(n T_{e}\right)=K_{\nu} I(t)-(3 / 2) n\left[\left(T_{e}-T_{i}\right) / \tau_{e}\right]-\beta n^{2} T_{e}^{1 / 2}, \\
& (3 / 2)(d / d t)\left(n T_{i}\right)=(3 n / 2)\left[\left(T_{e}-T_{i}\right) / \tau_{e}\right] .
\end{aligned}
$$

These equations represent the energy balance in the electrons and ions, where $K_{\nu}$ and $\tau_{e}$ are the absorption coefficient of the laser radiation and the electron-ion equilibration time, given by

$$
\begin{aligned}
& K_{\nu}=\alpha n^{2} / T_{e}^{3 / 2}, \\
& \tau_{e}=\gamma T_{e}^{3 / 2} / n .
\end{aligned}
$$

The numerical coefficients $\alpha, \beta, \gamma$ are explicitly given by

$$
\begin{aligned}
& \alpha=8 K \pi e^{6} / c \nu^{2}\left(2 \pi m_{e}\right)^{3 / 2}, \\
& \beta=32 \pi g(2 \pi)^{1 / 2} e^{6} / 3^{3 / 2} m_{e}^{3 / 2} c^{3} h, \\
& \gamma^{-1}=8(2 \pi)^{1 / 2} e^{4} m_{e}^{1 / 2} \log \Lambda / 3 m_{i} .
\end{aligned}
$$

In Eq. (5), $K$ is a constant of the order $10, m_{e}$ and $m_{i}$ are the electron and ion masses, $h$ is the Planck's constant, $c$ is the speed of light, $e$ is the electron charge, $\nu$ is the frequency of the laser light, $g$ is a quantum mechanical correction factor, and finally $\log \Lambda$ is the Coulomb logarithm.

In order to apply the methods of optimal control theory, we should simplify the notation in Eqs. (1) and (2). Thus, substituting the coefficients $K_{\nu}$ and $\tau_{e}$, from (3) and (4), in Eqs. (1) and (2), and defining the new variables $Z_{1}, Z_{2}$ as

$$
Z_{1} \equiv T_{e}, \quad Z_{2} \equiv T_{i}, \quad U(t) \equiv I(t),
$$

where $Z_{1}$ and $Z_{2}$ are referred to as the state variables and $U(t)$ as the control variable, the equations describing the laser heating of a plasma are then reduced to

$$
\begin{aligned}
& (d / d t) Z_{1}=A_{1} U(t) / Z_{1}^{3 / 2}-A_{2}\left(Z_{1}-Z_{2}\right) / Z_{1}^{3 / 2}-A_{3} Z_{1}^{1 / 2}, \\
& (d / d t) Z_{2}=A_{2}\left(Z_{1}-Z_{2}\right) / Z_{1}^{3 / 2},
\end{aligned}
$$

where the coefficients $A_{1}, A_{2}, A_{3}$ are defined as

$$
A_{1}=(2 / 3) \alpha n, \quad A_{2}=n / \gamma, \quad A_{3}=(2 / 3) \beta n .
$$

Equations (9) and (10) are referred to as the equations of state or plant equations. This system of equations will represent our mathematical model of the laser-plasma interaction. 
For the purpose of illustrating the application of the optimal control theory in this problem, we will consider a plasma composed of ordinary hydrogen ions and electrons and heated by a $\mathrm{CO}_{2}$ laser beam of maximum intensity $I_{0}=2.25 \times 10^{30} \mathrm{eV} / \mathrm{cm}^{2} \mathrm{~s}$, where the density $n$ is $2 \times 10^{17} / \mathrm{cm}^{3}$. Under these conditions the numerical values of the parameters are

$$
\begin{aligned}
& A_{1}=2.667 \times 10^{-17} \mathrm{eV}^{3 / 2} \mathrm{~cm}^{2}, \quad A_{2}=6.349 \times 10^{9} \mathrm{eV}^{3 / 2} / \mathrm{s}, \\
& \mathrm{A}_{3}=1.404 \times 10^{4} \mathrm{eV} / \mathrm{s} .
\end{aligned}
$$

\section{Optimal Control Formulation}

3.1. Hamilton's Equations. The optimal control problem can be formulated as follows: From all the control values that satisfy $0 \leq U(t) \leq I_{0}$, find the control strategy $U^{\text {op }}(t)$ which minimize the functional

$$
J=\int_{0}^{T}\left[C_{1}+C_{2} U(t)\right] d t
$$

and brings the system, through Eqs. (9) and (10), from an initial state

$$
Z_{1}(0)=T_{e 0}, \quad Z_{2}(0)=T_{i 0},
$$

to a final state with specified ion temperature $T_{i f}$; this condition on the final state can also be expressed as

$$
M\left(Z_{1}, Z_{2}\right)=Z_{2}-T_{i f}=0 .
$$

The final time $T$, which is the heating time, and the final electron temperature are not specified. The necessary conditions for optimality require the use of the Hamiltonian $H$, made up of the state variable $Z_{1}, Z_{2}$ and the adjoint variables or Lagrange multipliers $\lambda_{1}, \lambda_{2}$ as follows:

$$
\begin{aligned}
H & =C_{1}+C_{2} U+\lambda_{1}\left[A_{1} U / Z_{1}^{3 / 2}-A_{2}\left(Z_{1}-Z_{2}\right) / Z_{1}^{3 / 2}-A_{3} Z_{1}^{1 / 2}\right] \\
& +\lambda_{2} A_{2}\left[\left(Z_{1}-Z_{2}\right) / Z_{1}^{3 / 2}\right] .
\end{aligned}
$$

The optimal solution should satisfy the following equations (Ref. 4):

$$
\begin{aligned}
(d / d t) Z_{1} & =A_{1} U(t) / Z_{1}^{3 / 2}-A_{2}\left(Z_{1}-Z_{2}\right) / Z_{1}^{3 / 2}-A_{3} Z_{1}^{1 / 2}, \\
(d / d t) Z_{2} & =A_{2}\left(Z_{1}-Z_{2}\right) / Z_{1}^{3 / 2} \\
(d / d t) \lambda_{1} & =\left(\lambda_{1} / 2 Z_{1}^{5 / 2}\right)\left(3 A_{1} U-A_{2} Z_{1}+3 A_{2} Z_{2}+A_{3} Z_{1}^{2}\right) \\
& +\left(\lambda_{2} / 2 Z_{1}^{5 / 2}\right) A_{2}\left(Z_{1}-3 Z_{2}\right), \\
(d / d t) \lambda_{2} & =\left(A_{2} / Z_{1}^{3 / 2}\right)\left(\lambda_{2}-\lambda_{1}\right) .
\end{aligned}
$$


The optimal control is obtained from

$$
U^{\mathrm{op}}(t)= \begin{cases}I_{0}, & \text { if } C_{2}+A_{1} \lambda_{1} / Z_{1}^{3 / 2}<0, \\ 0, & \text { if } C_{2}+A_{1} \lambda_{1} / Z_{1}^{3 / 2}>0, \\ U_{s}(t), & \text { if } C_{2}+A_{1} \lambda_{1} / Z_{1}^{3 / 2}=0,\end{cases}
$$

where $U_{s}$ is the singular control (Ref. 8), to be discussed below. The transversality conditions (Ref. 4) for this problem are

$$
\begin{array}{ll}
\left.H(\bar{Z}, \bar{\lambda})\right|_{T}=0, & \\
\lambda_{1}(T)=0, & \\
\lambda_{2}(T)=\xi, & \xi \text { free, } \\
Z_{2}(T)=T_{i f}, & T_{\text {if }} \text { fixed, }
\end{array}
$$

where $\bar{Z}$ and $\vec{\lambda}$ are vectors with components $Z_{1}, Z_{2}$ and $\lambda_{1}, \lambda_{2}$, respectively. The initial conditions are given by Eqs. (14).

In addition, the Hamiltonian is a constant of motion along the optimal trajectory, because it is not an explicit function of time (Ref. 4). Hence, we have

$$
H(\bar{Z}, \bar{\lambda})=0, \quad 0 \leq t \leq T .
$$

The set of Eqs. (16)-(23) constitutes a two-point boundary-value problem involving nonlinear differential equations. Thus, it is impossible to obtain analytical solutions. However, the analysis that we present here enables us to obtain the necessary conditions that optimal trajectories must satisfy in order to minimize the prescribed functional. From these conditions, we will generate the so called switching curves in the phase plane $Z_{1}-Z_{2}$, that determine the optimal control switching points. These curves will be obtained numerically by flooding the phase plane with optimal backward trajectories that start on the target curve. The switching curves depend, as will be shown later, on the final ion temperature as well as on the ratio $C_{1} / C_{2}$ of the weighting parameters, but they are independent of the initial state of the system. This fact will permit us to obtain the optimal control law in terms of the instantaneous position of the state $\left(Z_{1}, Z_{2}\right)$ in the phase plane, regardless of whether it is an initial state or an intermediate state.

Candidates to optimal trajectories have to satisfy the above conditions. However, in order to isolate a unique optimal candidate, is necessary to analyze the possible existence and optimality of singular subarcs (Refs. 8 and 9). 
3.2. Singular Subarcs Candidates. Following Gabasov and Kirillova (Ref. 8), we divide the Hamiltonian in two parts,

$$
H=H_{0}(\bar{Z}, \bar{\lambda})+H_{1}(\bar{Z}, \bar{\lambda}) U
$$

where

$$
\begin{aligned}
& H_{0}=C_{1}+\left(\lambda_{1} / Z_{1}^{3 / 2}\right)\left(A_{2} Z_{2}-A_{2} Z_{1}-A_{3} Z_{1}^{2}\right)+\left(\lambda_{2} / Z_{1}^{3 / 2}\right)\left(A_{2} Z_{1}-A_{2} Z_{2}\right) \\
& H_{1}=C_{2}+A_{1} \lambda_{1} / Z_{1}^{3 / 2}
\end{aligned}
$$

The singular subarc may exist if the switching function $H_{1}$ vanishes in an interval of time of positive length. If the phase space trajectory is to satisfy this condition, then the higher-order total time derivatives of $H_{1}$ should also vanish. It can be easily shown that these derivatives of $H_{1}$ can be written as

$$
\left(d^{m} / d t^{m}\right) H_{1}=\alpha_{m}(\bar{Z}, \bar{\lambda})+\beta_{m}(\bar{Z}, \bar{\lambda}) U, \quad m=0,1, \ldots, 2 q
$$

this process continues until, for some integer number $q$, the coefficient $\beta_{2 q}$ does not vanishes identically. The singular control problem is said to be of order $q$.

In our problem, $\alpha_{m}$ and $\beta_{m}$ are obtained using (26) as

$$
\begin{aligned}
\alpha_{0}= & C_{2}+A_{1} \lambda_{1} / Z_{1}^{3 / 2}, \\
\beta_{0} \equiv & 0, \\
\alpha_{1}= & \left(A_{1} / 2 Z_{1}^{4}\right)\left(2 A_{2} \lambda_{1} Z_{1}+4 A_{3} \lambda_{1} Z_{1}^{2}+A_{2} \lambda_{2} Z_{1}-3 A_{2} \lambda_{2} Z_{2}\right) \\
\beta_{1} \equiv & 0 \\
\alpha_{2}= & -\left(4 \alpha_{1} / Z_{1}^{5 / 2}\right)\left(-A_{2} Z_{1}+A_{2} Z_{2}-A_{3} Z_{1}^{2}\right) \\
& +\left(A_{1} / 2 Z_{1}^{11 / 2}\right)\left(8 A_{2}^{2} \lambda_{1} Z_{2}-4 A_{2}^{2} \lambda_{1} Z_{1}-11 A_{2} A_{3} \lambda_{1} Z_{1}^{2}\right. \\
& -2 A_{2}^{2} \lambda_{2} Z_{1}+2 A_{2}^{2} \lambda_{2} Z_{2}+14 A_{2} A_{3} \lambda_{1} Z_{2} Z_{1} \\
& \left.-6 A_{3}^{2} \lambda_{1} Z_{1}^{3}+A_{2} A_{3} \lambda_{2} Z_{1}^{2}-6 A_{2} A_{3} \lambda_{2} Z_{2} Z_{1}\right) \\
\beta_{2}= & -4 \alpha_{1} A_{1} / Z_{1}^{5 / 2}+\left(A_{1}^{2} / 2 Z_{1}^{11 / 2}\right)\left(5 A_{2} \lambda_{1}+A_{2} \lambda_{2}+14 A_{3} \lambda_{1} Z_{1}\right) .
\end{aligned}
$$

Since $\beta_{2}$ does not vanish identically, we have a singular problem of order 1. By requiring that, on the singular arc, the switching function is to remain equal to zero in an interval of time of positive length, the singular arc is then specified by

$$
\begin{aligned}
& C_{2}+A_{1} \lambda_{1} / Z_{1}^{3 / 2}=0 \\
& 2 A_{2} \lambda_{1} Z_{1}+4 A_{3} \lambda_{1} Z_{1}^{2}+A_{2} \lambda_{2} Z_{1}-3 A_{2} Z_{2} \lambda_{2}=0
\end{aligned}
$$


To obtain the singular control we shall solve first the above equations for $\lambda_{1}$ and $\lambda_{2}$, as follows:

$$
\begin{aligned}
& \lambda_{1}=-C_{2} Z_{1}^{3 / 2} / A_{1}, \\
& \lambda_{2}=\left(2 C_{2} Z_{1}^{5 / 2} / A_{1} A_{2}\right)\left[\left(A_{2}+2 A_{3} Z_{1}\right) /\left(Z_{1}-3 Z_{2}\right)\right], \quad Z_{1} \neq 3 Z_{2} .
\end{aligned}
$$

Substituting now the above equations in the equations for $\alpha_{2}$ and $\beta_{2}$, the singular control is obtained by requiring the second-order time derivative of the switching function to be equal to zero. Thus, we have

$$
\begin{aligned}
U_{s} & =\left[24 A_{2}^{2}\left(Z_{1}-Z_{2}\right) Z_{2}+2 A_{3}^{2} Z_{1}^{3}\left(21 Z_{2}-5 Z_{1}\right)\right. \\
& \left.+A_{2} A_{3} Z_{1}\left(67 Z_{2} Z_{1}-42 Z_{2}^{2}-5 Z_{1}^{2}\right)\right] / \\
& \times\left[A_{1}\left(15 A_{2} Z_{2}-3 A_{2} Z_{1}-10 A_{3} Z_{1}^{2}+42 A_{3} Z_{1} Z_{2}\right)\right] .
\end{aligned}
$$

The above equation yields the general rule for the singular control law, which is not explicitly dependent on the weighting parameters $C_{1}$ and $C_{2}$; nevertheless, the implicit dependence lies on the fact that, as will be shown later, the state $\left(Z_{1}, Z_{2}\right)$ should lie on the singular arc, and this depends on the ratio $C_{1} / C_{2}$.

3.3. Optimality of the Singular Subarcs. In order to be considered an optimal candidate, a singular subarc should satisfy the generalized Legendre-Clebsh (GLC) condition (Ref. 9). For a singular control problem of order 1 , this condition reduces to

$$
(\partial / \partial U)\left(d^{2} H_{1} / d t^{2}\right) \leq 0
$$

or

$$
\beta_{2}(\bar{Z}, \bar{\lambda}) \leq 0
$$

To determine if the singular subarc satisfies the GLC condition (39), we will use the fact that the Hamiltonian is a constant of motion along trajectories that satisfy the necessary conditions for optimality. Using (16), (23), and (36), we obtain the following alternative expression for $\lambda_{2}$ along singular subarcs:

$$
\lambda_{2}=\left[C_{2} Z_{1}^{3 / 2}\left(A_{2} Z_{2}-A_{2} Z_{1}-A_{3} Z_{1}^{2}\right)-A_{1} C_{1} Z_{1}^{3 / 2}\right] /\left[A_{1} A_{2}\left(Z_{1}-Z_{2}\right)\right]
$$

Substituting the above expression together with (34) and (35) in the corresponding expression for $\beta_{2}$ in (33), the GLC condition becomes

$$
3 A_{2} C_{2}+\left(A_{3} C_{2} Z_{1}^{2}+A_{1} C_{1}\right) / 2\left(Z_{1}-Z_{2}\right)+7 A_{3} Z_{1} C_{2} \geq 0
$$

It is obvious from this expression that, in the region of the phase plane where the ion heating takes place (i.e., $Z_{1}>Z_{2}$ ), the GLC condition is always satisfied. 
3.4. Equation of the Singular Arc. The algebraic equation specifying the singular subarc is obtained by equating the expressions for $\lambda_{2}$ given in (37) and (40); thus, after few algebraic steps, we get

$$
\begin{aligned}
0 & =3 A_{2} C_{2} Z_{2}^{2}-\left[C_{2}\left(6 A_{2} Z_{1}+7 A_{3} Z_{1}^{2}\right)+3 A_{1} C_{1}\right] Z_{2} \\
& +3 A_{2} C_{2} Z_{1}^{2}+5 A_{3} C_{2} Z_{1}^{3}+A_{1} C_{1} Z_{1} .
\end{aligned}
$$

The above expression for the singular subarc depends on both $C_{1}$ and $C_{2}$, as expected.

We should point out here that, although this equation was obtained using (37), which requires $Z_{1} \neq 3 Z_{2}$, it reduces to the correct expression for the singular arc in the limiting cases of minimum time subarcs $\left(C_{2}=0\right.$, $\left.C_{1} \neq 0\right)$ and minimum energy subarcs $\left(C_{1}=0, C_{2} \neq 0\right)$, which are, respectively,

$0=Z_{1}-3 Z_{2} \quad$ (minimum time),

$0=3 A_{2} Z_{2}^{2}-\left(7 A_{3} Z_{1}^{2}+6 A_{2} Z_{1}\right) Z_{2}+3 A_{2} Z_{1}^{2}+5 A_{3} Z_{1}^{3}$ (minimum energy).

From the general expression for the singular subarcs, in (42), we can solve for $Z_{2}$ in the cases where $C_{2} \neq 0$. We obtain

$$
\begin{aligned}
Z_{2} & =Z_{1}+7 A_{3} Z_{1}^{2} / 6 A_{2}+A_{1} C_{1} / 2 A_{2} C_{2} \\
& -\left(1 / 6 A_{2}\right)\left[49 A_{3}^{2} Z_{1}^{4}+24 A_{2} A_{3} Z_{1}^{3}\right. \\
& \left.+\left(C_{1} / C_{2}\right)\left(42 A_{1} A_{3} Z_{1}^{2}+9 A_{1}^{2} C_{1} / C_{2}+24 A_{1} A_{2} Z_{1}\right)\right]^{1 / 2},
\end{aligned}
$$

where we have chosen the negative sign in the root square (in order for energy transfer from electrons to ions to exist, it is necessary that $Z_{1}>Z_{2}$ ).

It is possible to substitute $Z_{2}$ either from (43) or (45), depending on the particular case, in the equation for the singular control (38). The singular control then becomes only a function of the ratio $C_{1} / C_{2}$ and the electron temperature $Z_{1}$. For the particular case of minimum time trajectories $\left(C_{2}=0\right)$, the expression of the singular control reduces to

$$
U_{s}=\left(8 A_{2} Z_{1}+3 A_{3} Z_{1}^{2}\right) / 3 A_{1} \text {. }
$$

When $C_{2}=0$, the singular subarcs are specified by $Z_{1}=3 Z_{2}$. The latter has the following physical meaning: It is easily shown that the energy transfer rate, between Maxwellian electrons and ions given by the term $A_{2}\left(Z_{1}-Z_{2}\right) / Z_{1}^{3 / 2}$, is maximized when the corresponding temperatures satisfy the above relation.

Due to the constraint in the maximum laser intensity available [recall $U_{\min } \leq U(t) \leq U_{\max }$ with $U_{\min }=0$ and $U_{\max }=I_{0}$ ], there is always one state on the singular arc in which the singular control is equal to $U_{\max }$, i.e., the maximum value allowable. Thus, every singular trajectory has an upper 
bound which depends on the ratio $C_{1} / C_{2}$, called laser saturation state. The higher the maximum laser intensity available, the higher the upper bound is located.

Figure 2 shows different singular subarcs for several values of $C_{1} / C_{2}$. The values of the coefficients $A_{1}, A_{2}, A_{3}$ used to obtain these curves are given in Eq. (12).

As is expected, the behavior of singular arcs shows a transition between minimum time singular arcs, $Z_{1}=3 Z_{2}$, which maximize the energy transfer rate between electrons and ions, and minimum energy subarcs, which maximize the laser-electrons coupling, while minimizing the radiation losses. It should be pointed out that, if no radiation losses were included in the model, $A_{3}=0$, the minimum energy singular arcs degenerate into the $Z_{1}=Z_{2}$ straight line (the upper dashed curve). This is expected; when $Z_{1}=Z_{2}$, the coupling of the laser beam to the electrons is maximum, and the heating time is infinite, since the energy transfer rate between electrons and ions becomes infinitesimal.

3.5. Terminal and Control Switching Conditions. One can show, by analyzing the transversality conditions (22) and the behavior of the switching functions $H_{1}$, that the set of conditions to be satisfied at the final state and at the switching points can be summarized in three different cases as follows.

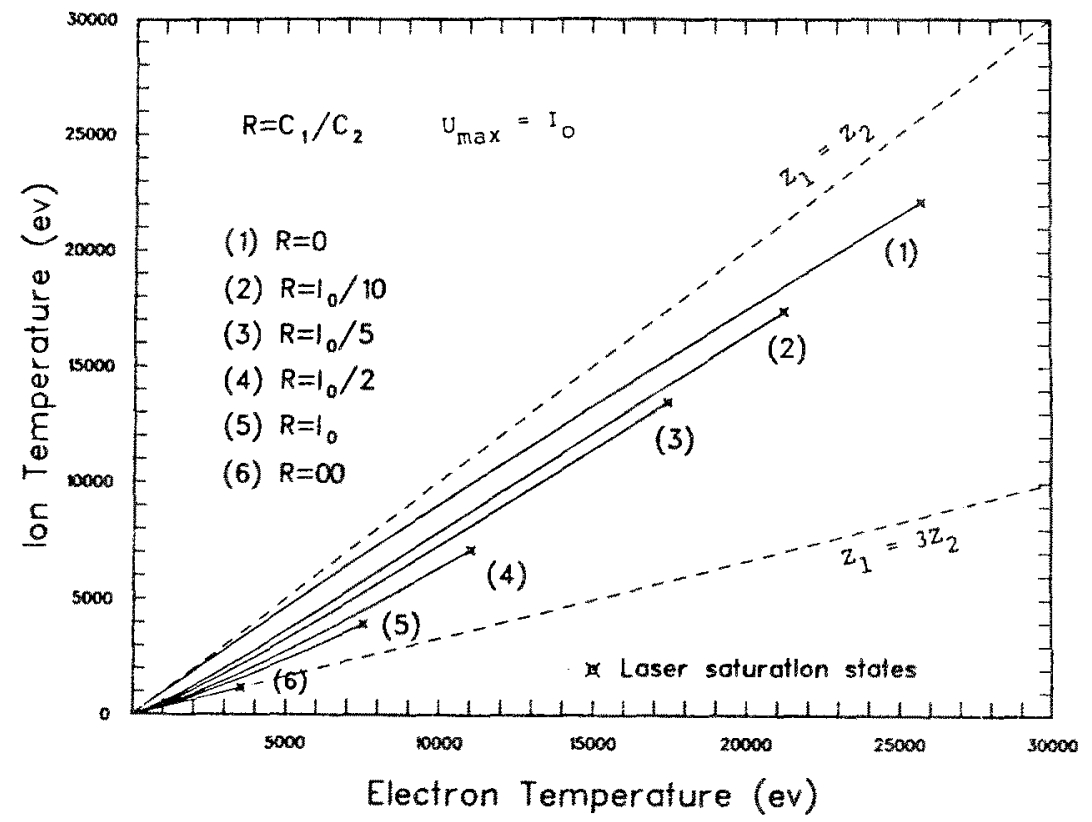

Fig. 2. Behavior of singular subarcs for several ratios of the weighting parameters. 
Case (i). Minimum Time Trajectories, $C_{1}=1, C_{2}=0$. The final state of a minimum time trajectory must satisfy the following conditions:

$$
\begin{aligned}
& Z_{1}(T)>Z_{2}(T), \\
& Z_{2}(T)=T_{i f}, \quad T_{i f} \text { fixed }, \\
& \lambda_{1}(T)=0, \\
& \lambda_{2}(T)=-\left[Z_{1}^{3 / 2} / A_{2}\left(Z_{1}-Z_{2}\right)\right]_{T} .
\end{aligned}
$$

The value of the optimal control at the final time $t=T$ can be found as follows ${ }^{4}$ :

$$
U^{\mathrm{op}}= \begin{cases}U_{\max }, & \text { if } Z_{1}(T)<3 Z_{2}(T) \\ 0, & \text { if } Z_{1}(T)>3 Z_{2}(T), \\ U_{s}, & \text { if } Z_{1}(T)=3 Z_{2}(T) \\ 0, & \text { if } Z_{1}(T)=3 Z_{2}(T)\end{cases}
$$

At a control switching time $t_{s}$, if any, the following conditions should hold:

$$
\begin{aligned}
& \lambda_{1}\left(t_{s}\right)=0, \\
& \lambda_{2}\left(t_{s}\right)=-\left[Z_{1}^{3 / 2} / A_{2}\left(Z_{1}-Z_{2}\right)\right]_{t_{s}} .
\end{aligned}
$$

The optimal control switching should be of the form

$$
\begin{aligned}
& U_{\max } \rightarrow U_{\min }, \quad \text { if } S<0, \\
& U_{\min } \rightarrow U_{\max }, \quad \text { if } S>0, \\
& U_{\max } \leftrightarrows \mathrm{U}_{\mathrm{s}} \text { or } U_{\min } \rightarrow U_{s}, \quad \text { if } S=0 .
\end{aligned}
$$

The singular function $S$ is given by

$$
S=Z_{1}-3 Z_{2}
$$

Case (ii). Minimum Energy Trajectories, $C_{1}=0, C_{2}=1$. At the final state, minimum energy trajectories should satisfy

$$
\begin{aligned}
& Z_{1}(T) \geq Z_{2}(T), \\
& Z_{2}(T)=T_{i f},
\end{aligned}
$$

\footnotetext{
${ }^{4}$ Equation (51c) holds, provided this state lies at or below the laser saturation state. Equation (51d) holds, provided this state lies above the laser saturation state.
} 


$$
\begin{aligned}
& \lambda_{1}(T)=0, \\
& \lambda_{2}(T)= \begin{cases}0, & \text { if } Z_{1}(T)>Z_{2}(T), \\
\text { free, } & \text { if } Z_{1}(T)=Z_{2}(T)\end{cases}
\end{aligned}
$$

At the final state, the optimal control is always

$$
U^{\mathrm{OP}}(T)=0 \text {. }
$$

Here, any state on the $U_{\min }$ trajectory that crosses the state in which $Z_{1}(T)=Z_{2}(T)$ and lies above the singular arc, i.e., where $S<0$, may be a switching point. In all cases, the following conditions should be satisfied at the switching point:

$$
\begin{aligned}
& \lambda_{1}\left(t_{s}\right)=-\left(Z_{1}^{3 / 2} / A_{1}\right)_{t_{s}} \\
& \lambda_{2}\left(t_{s}\right)=\lambda_{1}\left(t_{s}\right)\left[1+A_{3} Z_{1}^{2} / A_{2}\left(Z_{1}-Z_{2}\right)\right]_{t_{s}}
\end{aligned}
$$

The optimal control switching, if any, should be of the form

$$
\begin{aligned}
& U_{\max } \rightarrow U_{\min }, \quad \text { if } S<0, \\
& U_{\min } \rightarrow U_{\max }, \quad \text { if } S>0, \\
& U_{\max } \rightleftarrows U_{s} \text { or } U_{\min } \rightleftarrows U_{s}, \quad \text { if } S=0 .
\end{aligned}
$$

The singular function in this case is given by

$$
S=3 A_{2} Z_{2}^{2}-\left(7 A_{3} Z_{1}^{2}+6 A_{2} Z_{1}\right) Z_{2}+3 A_{2} Z_{1}^{2}+5 A_{3} Z_{1}^{3}
$$

We should point out here that the value of $\lambda_{2}(T)$, which is not specified in (59), is to be chosen such that the conditions in (61) and (62) are satisfied.

Case (iii). General Case, $C_{1}>0, C_{2}>0$. At the final state, the corresponding optimal trajectories should satisfy the conditions

$$
\begin{aligned}
& Z_{1}(T)>Z_{2}(T), \\
& Z_{2}(T)=T_{i f} \\
& \lambda_{1}(T)=0, \\
& \lambda_{2}(T)=-\left[C_{1} Z_{1}^{3 / 2} / A_{2}\left(Z_{1}-Z_{2}\right)\right]_{T} .
\end{aligned}
$$

The final optimal control at any final state is given by

$$
U^{\mathrm{op}}(T)=0
$$


At the control switching time $t_{s}$, if any, the following conditions on the adjoint variables should hold:

$$
\begin{aligned}
& \lambda_{1}\left(t_{s}\right)=-\left(C_{2} Z_{1}^{3 / 2} / A_{1}\right)_{t_{s}} \\
& \lambda_{2}\left(t_{s}\right)=\lambda_{1}\left(t_{s}\right)\left[1+A_{3} Z_{1}^{2} / A_{2}\left(Z_{1}-Z_{2}\right)+A_{1} C_{1} / A_{2} C_{2}\left(Z_{1}-Z_{2}\right)\right]_{t_{s}} .
\end{aligned}
$$

The optimal control switching should be of the form

$$
\begin{aligned}
& U_{\max } \rightarrow U_{\min }, \quad \text { if } S<0, \\
& U_{\min } \rightarrow U_{\max }, \quad \text { if } S>0, \\
& U_{\max } \rightleftarrows U_{s} \text { or } U_{\min } \rightleftarrows U_{s}, \quad \text { if } S=0 .
\end{aligned}
$$

In this case, the function $S$ is given by

$$
\begin{aligned}
S\left(Z_{1}, Z_{2}, C_{1}, C_{2}\right) & =3 A_{2} C_{2} Z_{2}^{2}+3 A_{2} C_{2} Z_{1}^{2}+5 A_{3} C_{2} Z_{1}^{3}+A_{1} C_{1} Z_{1} \\
& -7 A_{3} C_{2} Z_{2} Z_{1}^{2}-3 A_{1} C_{1} Z_{2}-6 A_{2} C_{2} Z_{1} Z_{2} .
\end{aligned}
$$

3.6. Switching Curves. In this section, the necessary conditions that any optimal trajectory should satisfy at the final state were used to generate optimal backward trajectories, in order to find the switching curves, i.e., the set of points in the phase plane where the optimal control switching occurs. The backward trajectories start on the target curve. The switching points for each optimal backward trajectory are located where the switching conditions summarized in the last section are satisfied.

Each backward trajectory was obtained by numerical integration of Eqs. (17) through (20), using a simple corrector-predictor method.

The objective of generating the switching curves is to separate the phase plane into regions of well-defined optimal controls. In order to illustrate this, in Fig. 3 a final ion temperature of $5 \mathrm{keV}$ and a ratio of weighting parameters $C_{1} / C_{2}=5 U_{\max }$ were chosen, and optimal trajectories were obtained for different sets of initial conditions. The set of points where the optimal trajectories switch to a different optimal control is shown at the bottom of this figure (dashed lines). These set of points in the phase plane form the so called switching curves. The shape and location of the switching curves in the phase plane depend on the ratio of the weighting parameters $C_{1} / C_{2}$, as well as on the final ion temperature.

Figures 4 and 5 show switching curves corresponding to minimum time trajectories (i.e., when $C_{1}=1$ and $C_{2}=0$. It should be noticed that in this case, if the laser saturation state lies below the target line, all singular trajectories have an exit point on the singular arc that lies below the laser 

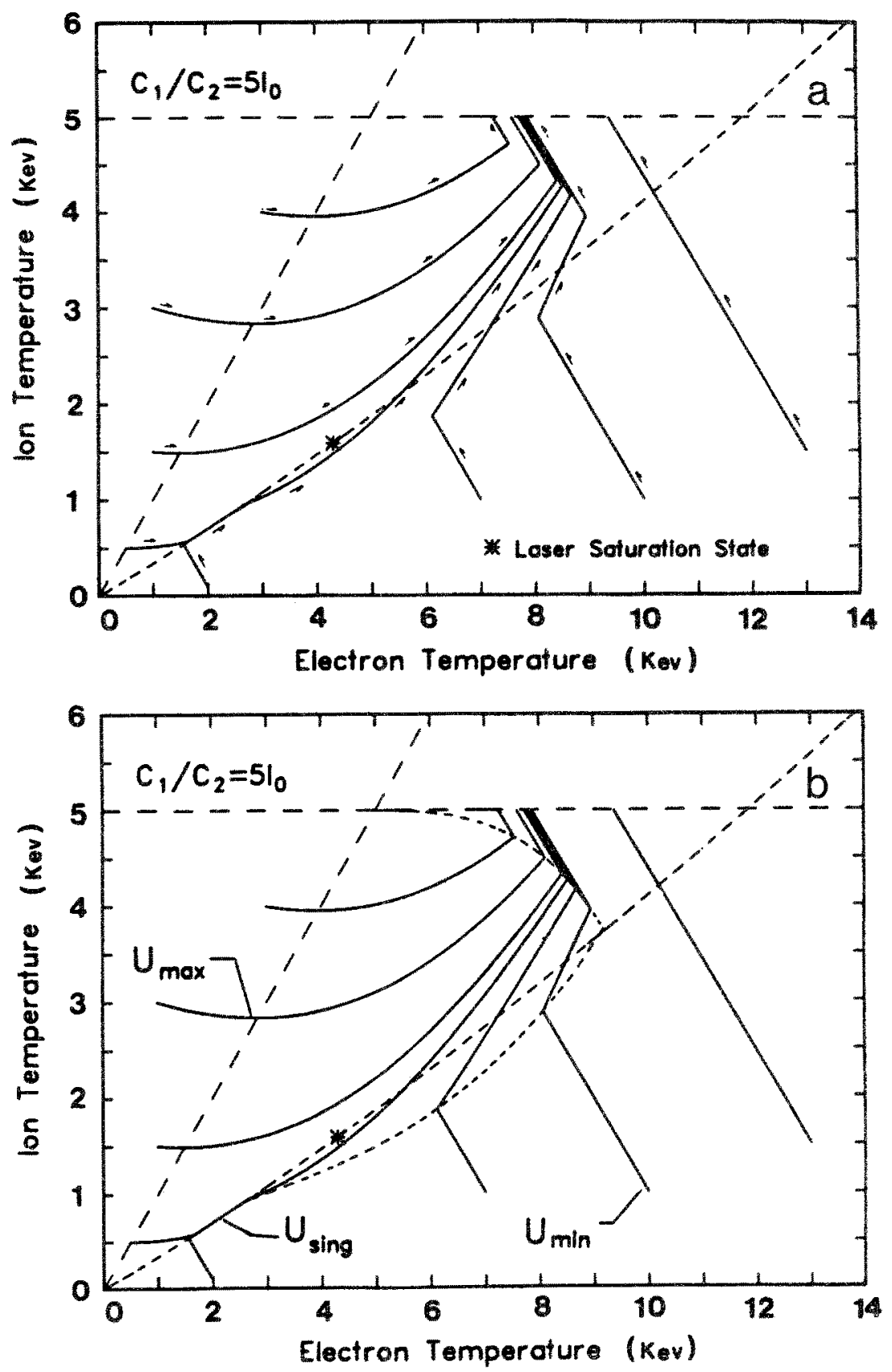

Fig. 3. Some optimal trajectories corresponding to $C_{1} / C_{2}=5 U_{\max }$ and a final ion temperature of $5 \mathrm{keV}$. 


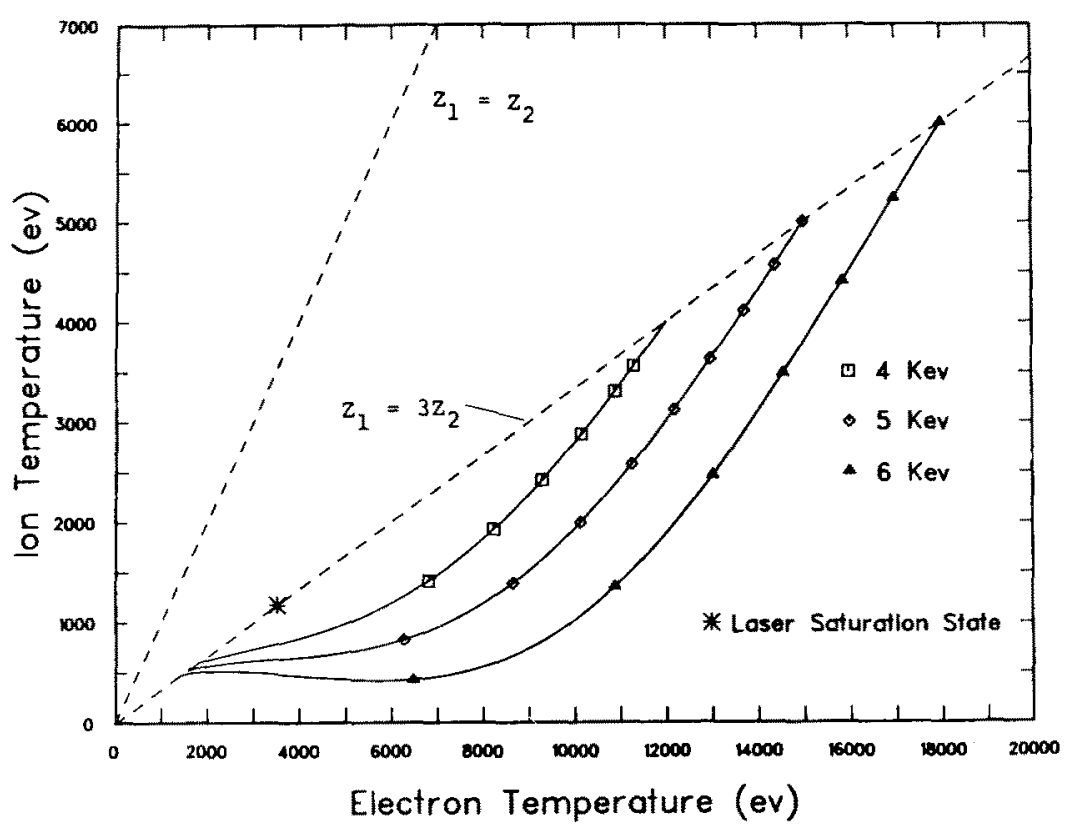

Fig. 4. Switching curves corresponding to minimum time trajectories for three different final ion temperatures.

saturation state and depends only on the final ion temperature. On the other hand, if the target line lies below the laser saturation state, then singular trajectories may be final optimal subarcs, as discussed before, and no bang-bang switching curves exist.

In Fig. 6, switching curves corresponding to minimum energy trajectories, $C_{1}=0$ and $C_{2}=1$, are shown for several final ion temperatures. For the particular final ion temperatures chosen in this figure, the laser saturation state lies always above the target line; thus, the exit point from the singular arc corresponds to the intersection point between the singular arc and the $U_{\min }$ trajectory leading to thermal equilibrium of ions and electrons at the desired final ion temperature.

Figure 7 shows the behavior of the switching curves corresponding to a ratio $C_{1} / C_{2} U_{\max }=1$, where $C_{1}$ and $C_{2} U_{\max }$ are both dimensionless, for several values of the final ion temperature. The solid line in this figure corresponds to the singular arc, and the curves marked with different temperature values are the switching curves corresponding to different final ion temperatures. We can observe in this figure that, in all cases, a switching curve located above the singular arc exists, which corresponds to $U_{\max } \rightarrow$ $U_{\min }$ transitions, and from now on it will be referred to as upper switching 


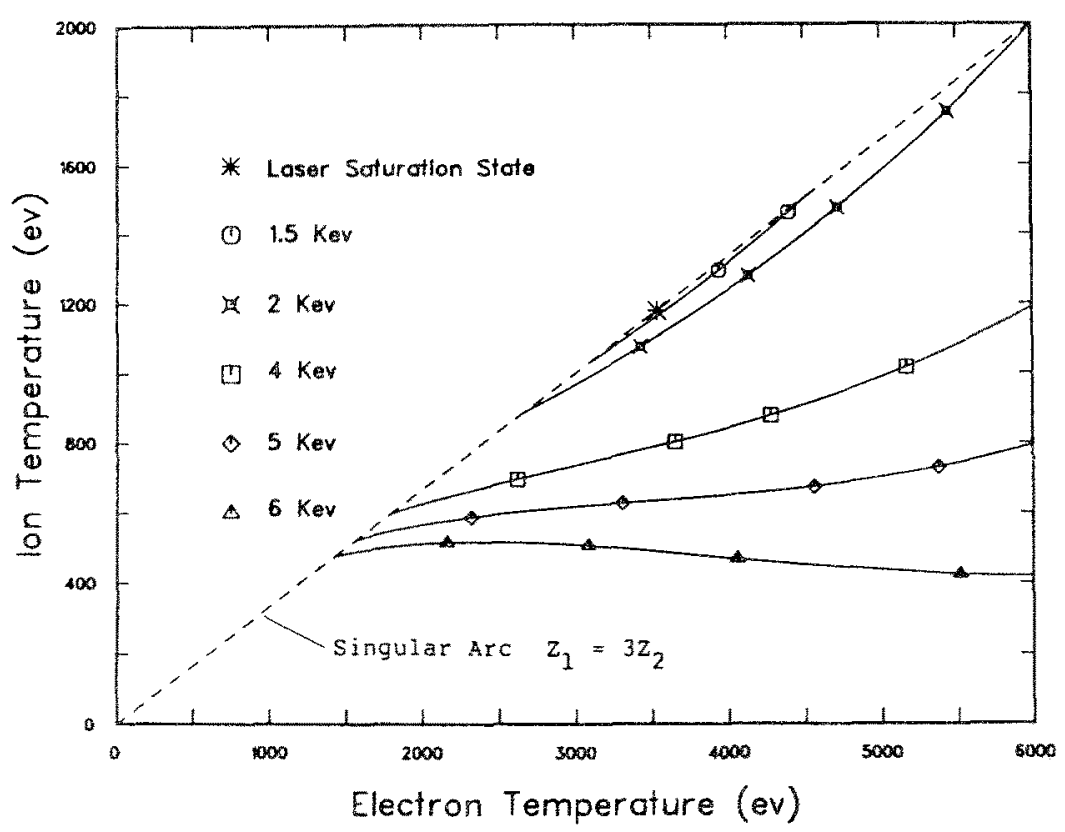

Fig. 5. Behavior of minimum time switching curves around the laser saturation state.

curve. When the upper switching curve intersects the singular arc above, the laser saturation state, another switching curve appears below the singular arc and corresponds to $U_{\min } \rightarrow U_{\max }$ transitions, referred to as lower switching curve. One of the extreme points of this latter switching curve is the intersection point between the singular arc and the upper switching curve; the other extreme lies somewhere on the singular arc below the laser saturation state.

We should point out that, in both cases, i.e., when only the upper switching curve exists or when both switching curves exist, singular trajectories contain always an exit point that lies below or at the laser saturation state and corresponds to transitions of the type $U_{s} \rightarrow U_{\min }$ in the first case or $U_{s} \rightarrow U_{\max }$ in the latter case. This characteristic is due to the fact that, when $C_{2}>0$, singular trajectories can never be optimal final subares.

In Fig. 8, a fixed final ion temperature of $5 \mathrm{keV}$ was chosen to show the behavior of the switching curves when the weighting parameters $C_{1}$ and $C_{2}$ vary between the limiting cases of minimum energy trajectories, $\left(C_{1}=0, C_{2}=1\right)$ and minimum time trajectories $\left(C_{1}=1, C_{2}=0\right)$.

The objective of the switching curves, as mentioned earlier, is to separate the phase plane into regions according to the optimal control associated with each point of the phase plane. In general, we can separate the problem 


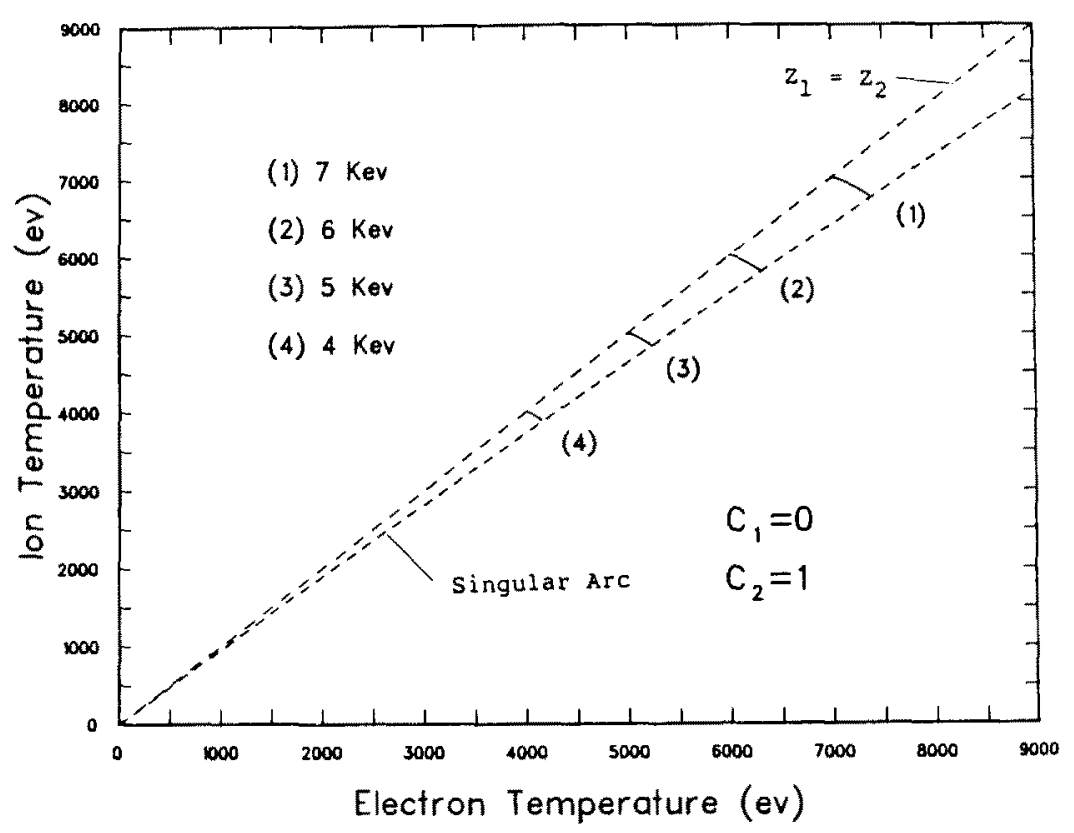

Fig. 6. Switching curves corresponding to minimum energy trajectories for several final ion temperatures.

of determining the optimal control in two cases, which are illustrated in Fig. 9. In the first case, only the upper switching curve exists; in the latter case, both the lower and upper switching curves exist.

When only the upper switching curve exists, the optimal control law can be specified as follows: If the state of the system is located in the region bounded by the singular arc, the upper switching curve, the $Z_{2}$-axis, and the $Z_{2}=T_{i f}$ straight line, then the optimal control is $U_{\max }$. If the state is located to the right-hand side of this region, the optimal control is $U_{\min }$. When the state of the system lies on the singular arc, in the boundary between these two regions, the optimal control is $U_{s}$.

When lower and upper switching curves exist, the optimal control law is specified as follows: If the state of the system lies in the region bounded by the singular arc, the lower and upper switching curves, the $Z_{2}=T_{i f}$ line, and the $Z_{2}$-axis, the optimal control is $U_{\max }$. If the state is located to the right-hand side of this zone, the optimal control is $U_{\min }$. When the state is located on the singular arc corresponding to the boundary between these two regions, the singular control is then optimal.

As we can notice, in both cases, if the state of the system is located in the region $Z_{1}<Z_{2}$, the optimal control is always $U_{\max }$. This is reasonable, 


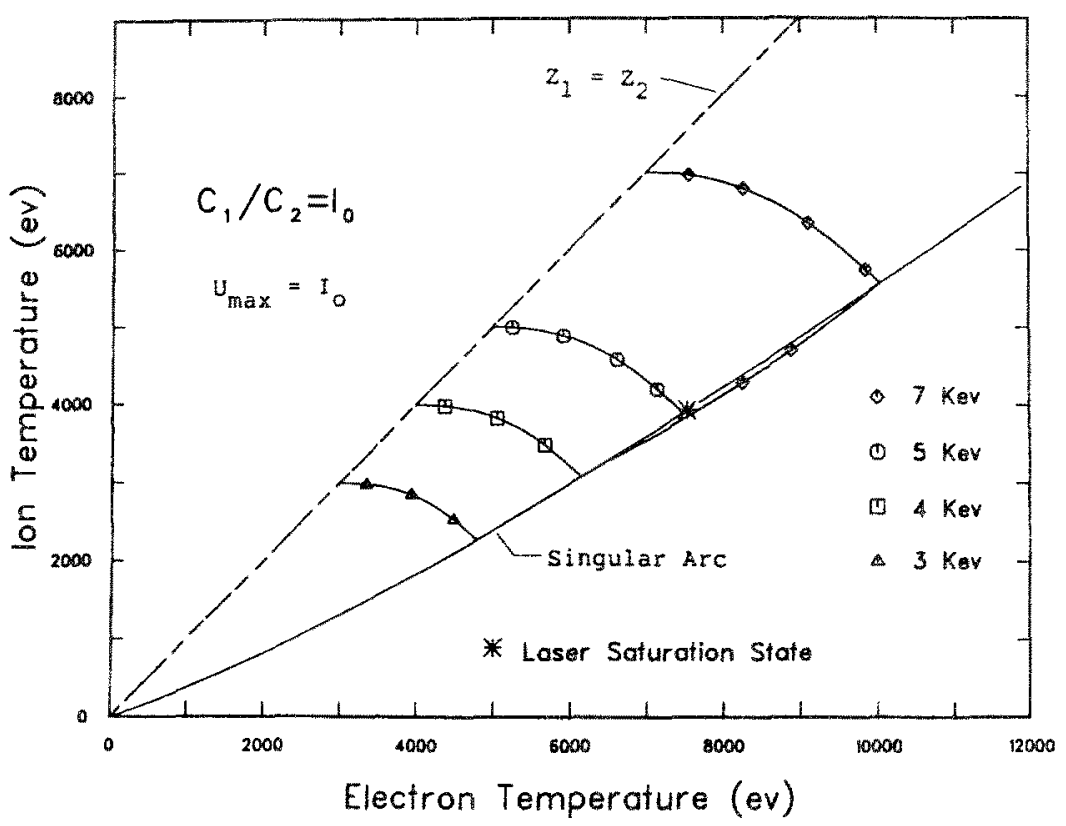

Fig. 7. Behavior of the switching curves corresponding to $C_{1} / C_{2}=U_{\max }$ for several final ion temperatures.

because in that region no ion heating occurs, and it is desirable to quickly heat the electrons in order to avoid energy transferred from the ions to the electrons, which is considered undesirable, since our objective is to heat the ions. On the other hand, if the electron temperature is larger that the ion temperature, it may be possible that the optimal heating strategy is to leave the electron and ion temperature to relax, i.e., with $U^{\mathrm{op}}=0$.

In the next section, the implementation of the optimal control strategies described above and the way how optimal switching curves should be used for this purpose will be illustrated.

\section{Optimal Trajectories}

In this section, typical optimal trajectories for several ratios $C_{1} / C_{2}$ of the weighting parameters are obtained following the optimal control strategy presented in the last section. An initial state corresponding to a temperature of $10 \mathrm{eV}$ for both ions and electrons and a final ion temperature of $5 \mathrm{keV}$ was used in all of the following cases, in order to compare the total energy spent and the heating time in the process, when different sets of weighting 


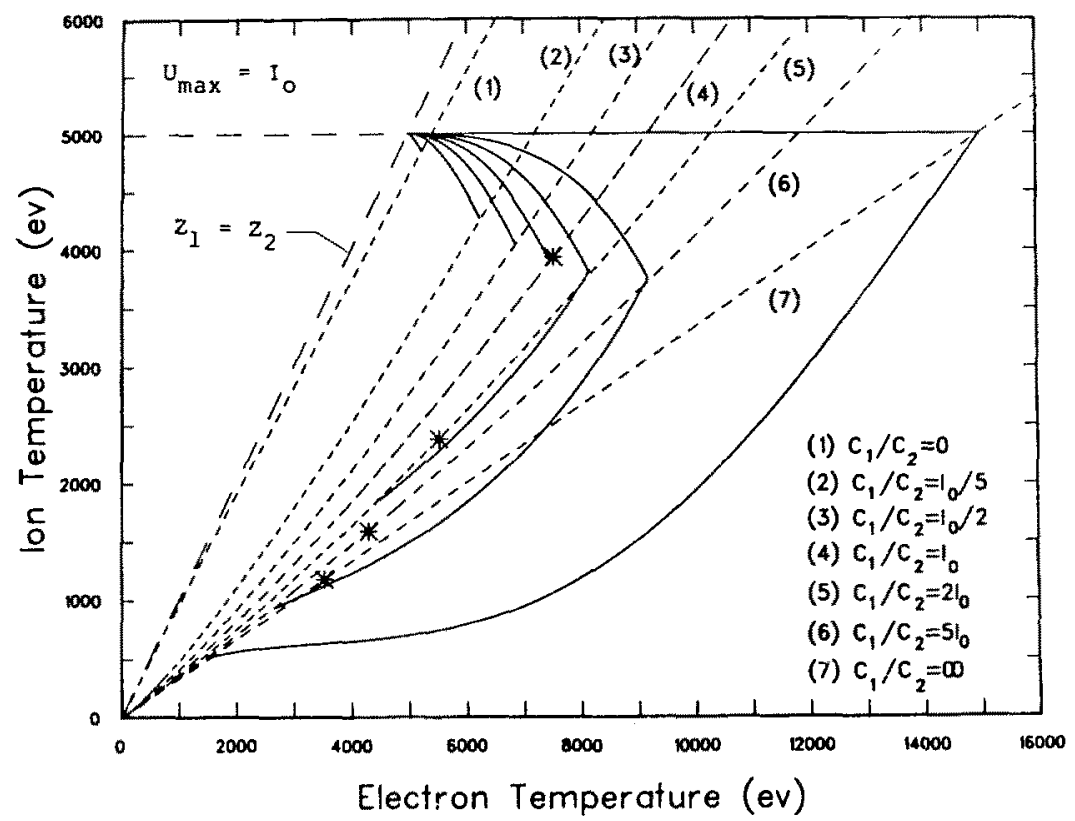

Fig. 8. Behavior of switching curves (continuous lines), given the final ion temperature of $5 \mathrm{keV}$, for several values of $C_{1} / C_{2}$. The dashed lines correspond to singular ares.

parameters are used. The set of weighting parameters that were used corresponds to those utilized to obtain the switching curves in Fig. 8.

When the optimal control strategies discussed in the last section were implemented, the optimal trajectories in the phase plane shown in Fig. 10 were obtained. We can observe that, due to the choice of the initial state, all the trajectories contain a singular subarc. Initially the optimal control in each of the optimal trajectories is $U_{\max }$, until the corresponding singular subarc is reached; due to the smallness of these initial arcs, these cannot be observed in this figure.

As is expected, the tendency of the minimum energy trajectory is to keep the electron temperature as small as possible, because this increases the coupling between the laser beam and the electrons, while reducing the bremsstrahlung radiation. It should be pointed out once again that, if no bremsstrahlung radiation were included in the model, the singular arc for the case of minimum energy trajectories reduces to the straight line $Z_{1}=Z_{2}$. This is due to the fact that, since no energy losses exist, the heating time may grow indefinitely without affecting the penalty function; thus, reducing the electron temperature increases the efficiency in the absorption of the laser energy by the electrons. In this case, however, the energy transfer rate 

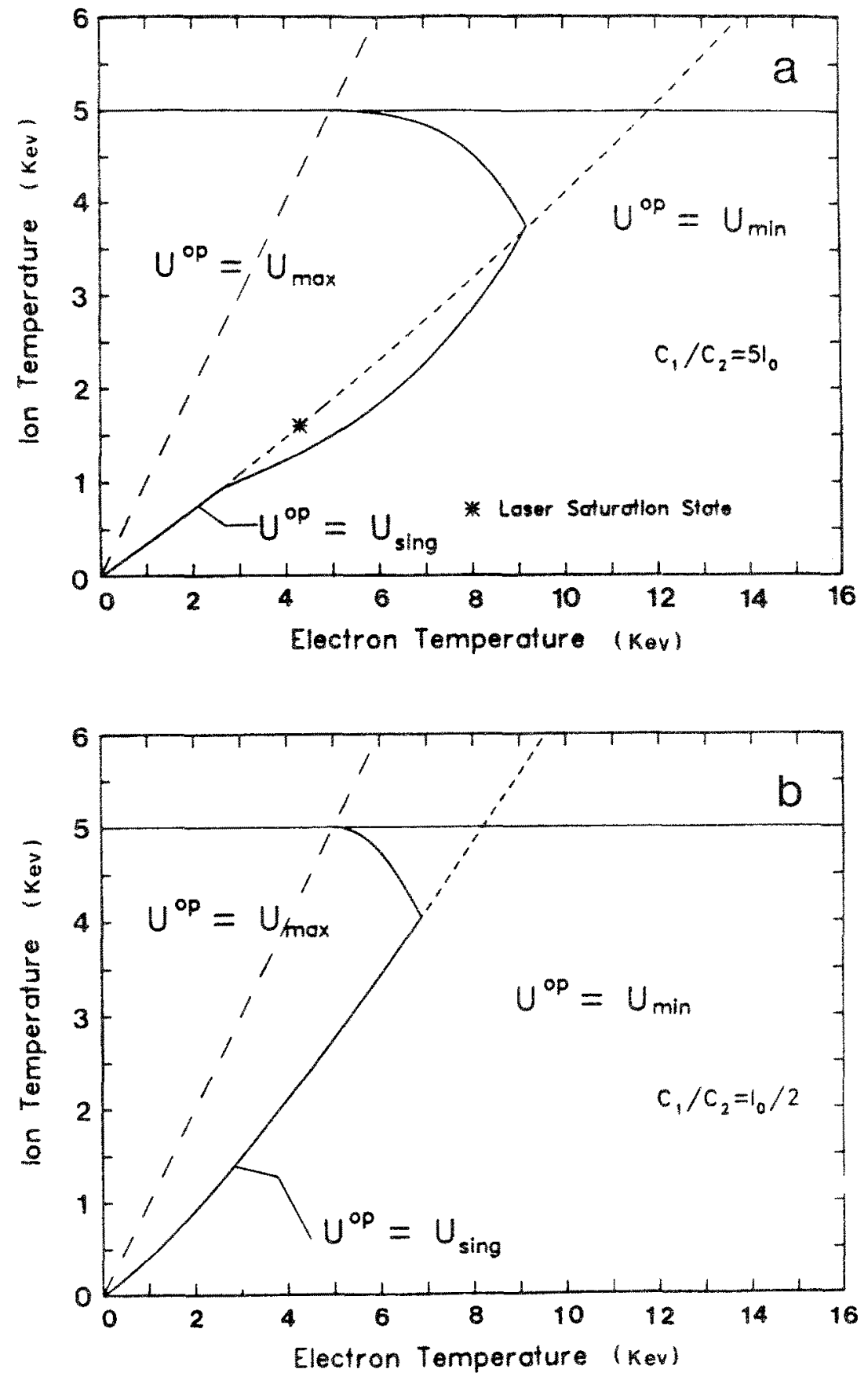

Fig. 9. Typical optimal control zones. 


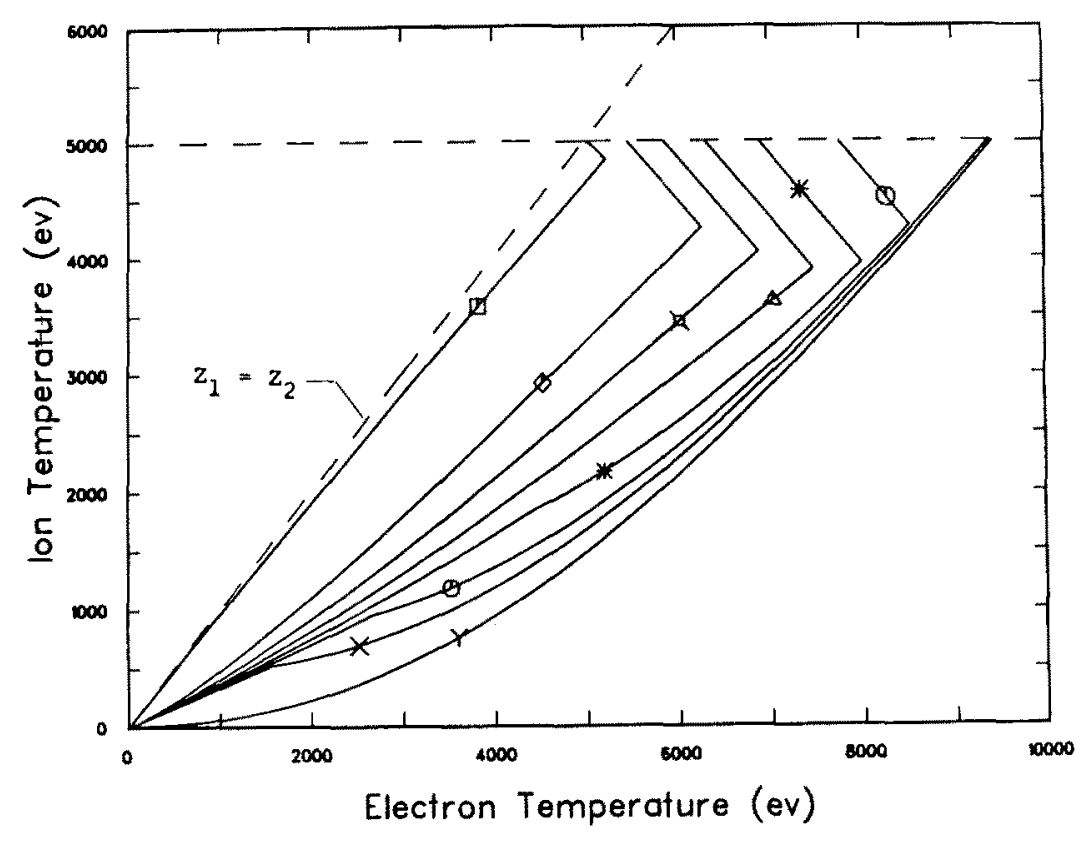

Fig. 10. Behavior of optimal trajectories for an initial state of $10 \mathrm{eV}$ (ions and electrons) and a final ion temperature of $5 \mathrm{keV} . \square R=0, \diamond R=1 / 5, \square(R=1 / 2,4 R=1, * R=2$, $O R=5, \times R=\infty, Y$ constant laser pulse. $\left.R=C_{1} / C_{1} / C_{2} I_{0}\right)$.

between electrons and ions in the plasma becomes infinitesimally small; consequently, the heating time becomes infinite, since [see Eq. (38)] the singular control becomes zero.

Minimum time trajectories, on the other hand, keep the electron temperature three times larger than the ion temperature along the singular subarc, since this condition maximizes the energy transfer rate from electrons to ions. The exit point from the singular arc appears below the laser saturation state, contrary to what we could have expected on the basis that the singular trajectory would be optimal all the way until the laser saturation state is reached. This is due to the fact that the $U_{\max }$ trajectory is tangent to the singular arc at the laser saturation state, so that the electron temperature is always smaller that three times the ion temperature after this point. Although the reduction in the electron temperature decreases the denominator of the energy transfer rate term, given by $A_{2}\left(Z_{1}-Z_{2}\right) / Z_{1}^{3 / 2}$, the reduction of the electron and ion temperature differences overwhelms this effect, yielding in the long run an overall smaller energy transfer efficiency. This explains why the exit point lies below the laser saturation 
JOTA: VOL. 52, NO. 1, JANUARY 1987
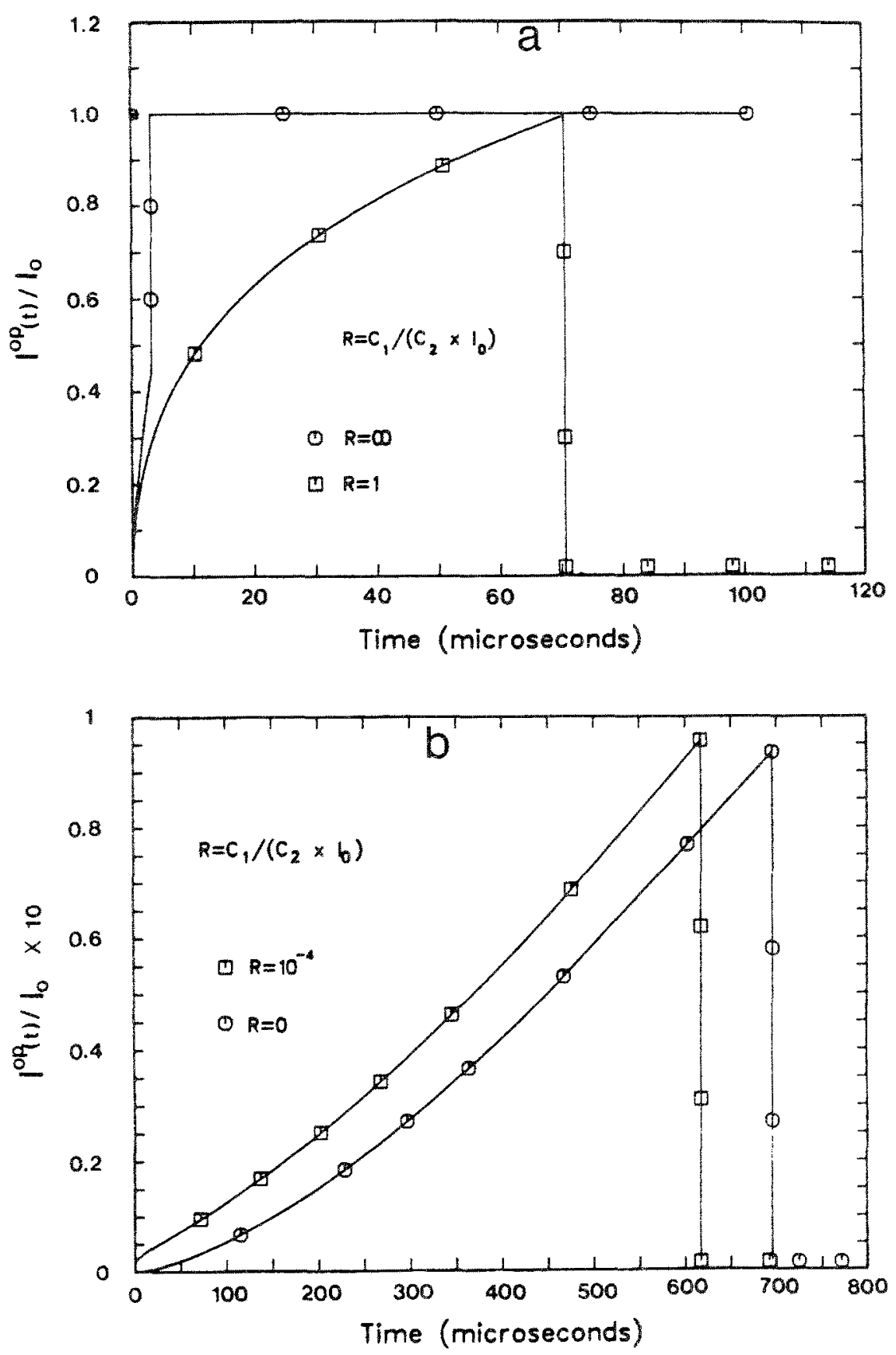

Fig. 11. Optimal laser pulses for several ratios of the weighting parameters. 

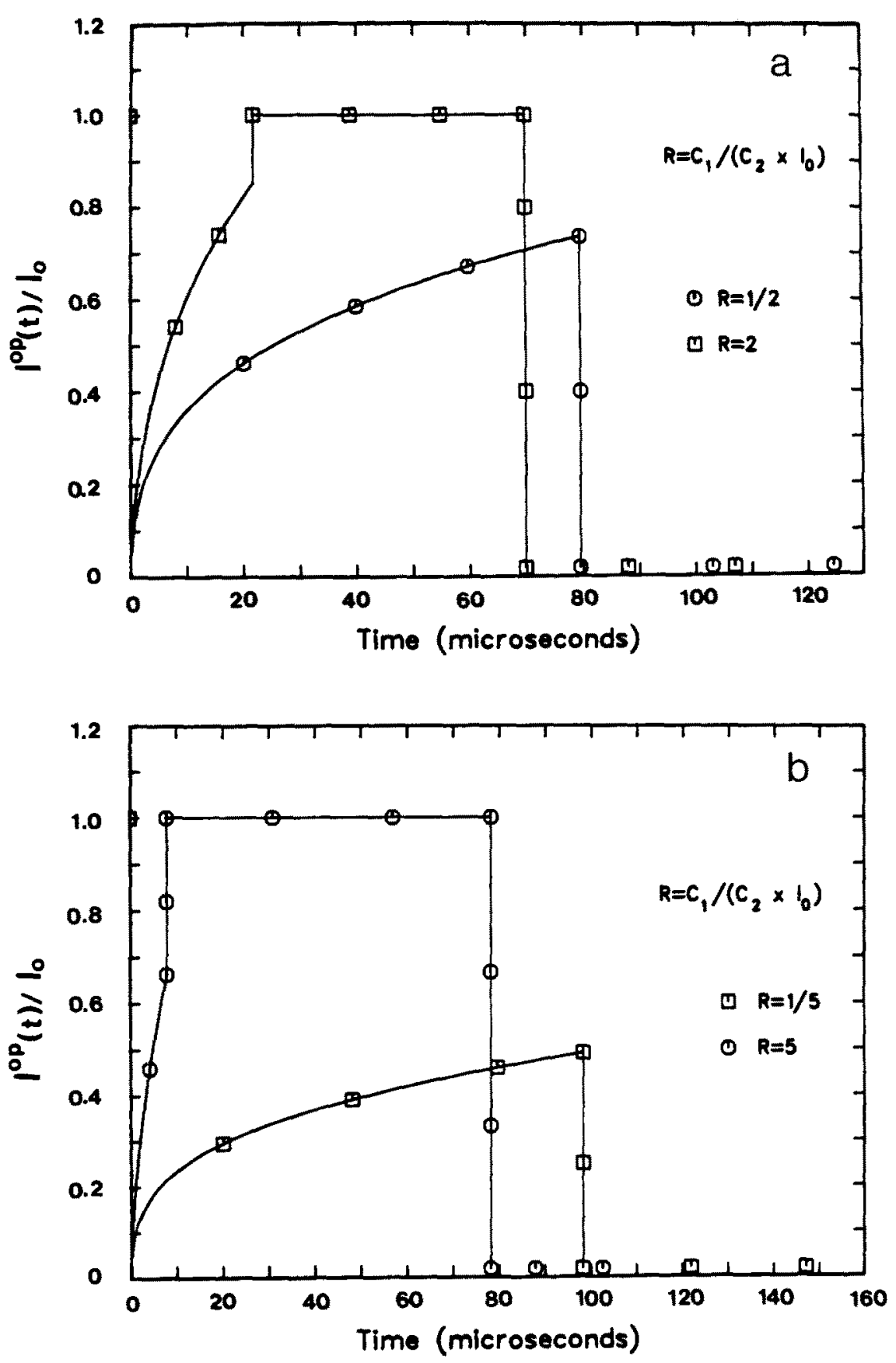

Fig. 12. Optimal laser pulses for several ratios of the weighting parameters. 

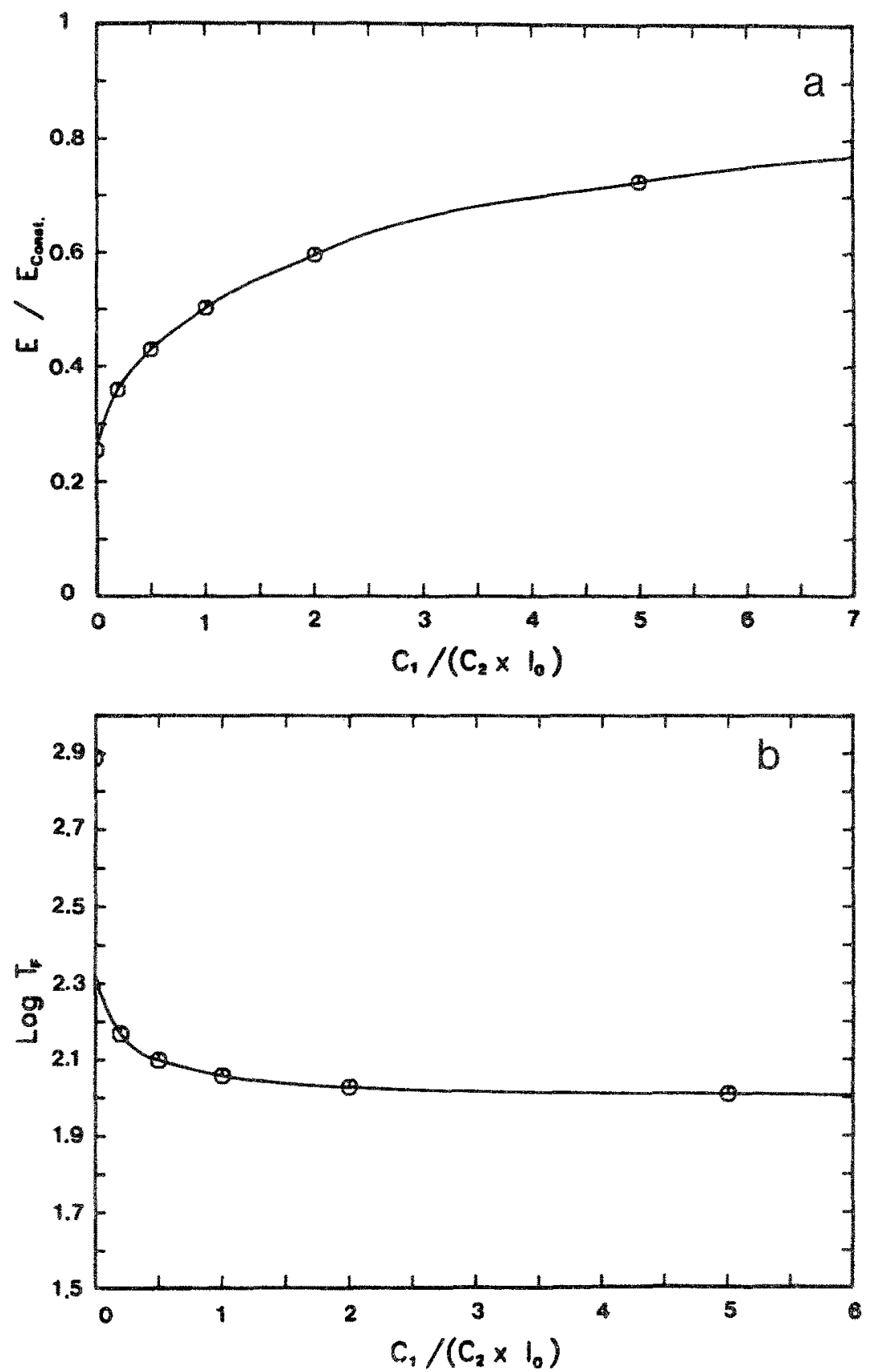

Fig. 13. Fraction of the energy spent using a constant laser pulse and logarithm of the heating time (in microseconds) versus ratio of the weighting parameters. 
state. The exact exit state is determined by the final conditions, in this case by the final ion temperature. These features are depicted in Figs. 3 and 4.

The other trajectories constitute a compromise between the energy spent and the heating time, for different weighting coefficients.

The optimal laser intensity profiles that drive the plasma from the initial state to the final state along the optimal trajectories shown in Fig. 10 are plotted as a function of time in Figs. 11 and 12.

Table 1 shows the control switching times, the heating time, and the total energy spent in the heating process for the different ratios $C_{1} / C_{2}$ used in the calculations.

Figure 13 shows the behavior of the logarithm of the heating time in microseconds and the fraction of the energy spent in the heating process, relative to the energy needed when a constant laser pulse is utilized. Both are plotted as functions of the ratio $C_{1} / C_{2} U_{\max }$. A possible use of these results may be demonstrated as follows. Suppose that the initial state of the plasma corresponds to an equilibrium state at $10 \mathrm{eV}$, for both electrons and ion, and that the maximum energy available $E_{m}$ and the maximum laser intensity $I_{0}$ have been given for the laser system being used. Then, the maximum value of the ratio $C_{1} / C_{2}$ can be determined from Fig. 13 with the given $E_{m} / E_{\text {const }}$. This value of $C_{1} / C_{2}$ yields the minimum heating time possible for the given total energy. If a smaller value of $C_{1} / C_{2}$ is chosen, compared with the above maximum, then the heating time will be larger; however, the energy spent in the heating process will be smaller.

Another possible use of Fig. 13 is that it allows one to determine the minimum energy necessary to heat the ions from the initial state to the desired final ion temperature. Thus, if there is a constraint in the total energy available, we can always determine whether it is enough to heat the ions to the desired temperature and, if so, we can find the most convenient heating strategy by choosing an appropriate value of the ratio $C_{1} / C_{2}$.

\section{Conclusions}

We have shown that, for plasmas of constant density, a significant reduction in energy spent in the heating process may be attained with only a slight increase in the heating time, when optimum laser intensity profiles are used, as compared to the total energy required when the maximum laser intensity is used to heat the plasma. This result is the primary motivation for resorting to the optimal control theory.

On the other hand, minimum time trajectories may not yield a significant reduction in heating time as compared with the use of a constant laser 
pulse, as can be seen by comparing the last two lines in the last two columns in Table 1.

The minimum energy necessary to heat the plasma ions to the desired temperature can be obtained by appropriately shaping the laser pulse, although this procedure yields relatively large heating times, which may not be reasonable if the plasma confinement time is smaller than the heating time obtained with the minimum energy trajectories.

The method described here can be applied to more realistic plasma models. An extension that includes the expansion of the plasma against the magnetic field, as well as the bremsstrahlung radiation losses, will be reported later.

\section{References}

1. LEE, Y. C., and KAW, P. K., Parametric Instabilities of Ion Cyclotron Waves in a Plasma, Physics of Fluids, Vol. 15, pp. 911-918, 1972.

2. Vagners, J., Neal, R. D., and Vlases, G. C., Optimal Laser Plasma Heating in a Solenoidal Magnetic Field, Physics of Fluids, Vol. 18, pp. 1314-1320, 1975.

3. Pontryagin, L. S., Boltyanskit, V. G., Gamkelide, R. V., and Mishchenko, E. F., The Mathematical Theory of Optimal Processes, John Wiley, New York, New York, 1962.

4. Bryson, A. E., JR., and Ho, Y. C., Applied Optimal Control, Hemisphere Publishing Company, New York, New York, 1975.

5. KRISTIANSEN, M., and HAGLER, M. O., Laser Heating of Magnetized Plasmas, Nuclear Fusion, Vol. 16, pp. 999-1034, 1976.

6. YUEN, S. Y., LAX, B., and COHN, D. R., Laser Heating of a Magnetically Confined Plasma. Physics of Fluids, Vol. 18, pp. 829-836, 1975.

7. DAwson, J, and OBERMAN, C., High-Frequency Conductivity and the Emission and Absorption Coefficients of a Fully Ionized Plasma, Physics of Fluids, Vol. 5 , pp. 517-524, 1962.

8. GaBASOV, R., and Kirillova, F. M., Higher-order Conditions for Optimality, SIAM Journal of Control, Vol. 10, pp. 127-168, 1972.

9. Kelley, H. J., KoOP, R. E., and MOYeR, A. G., Topics in Optimization, Vol. 2, Edited by G. Leitmann, Academic Press, New York, New York, 1966. 